\title{
Die Bearbeiter der 1. Auflage
}

Professor Dr. Diederich Eckardt, Professor an der Universität Trier

Professor Dr. Ulrich Ehricke, LL.M. (London), M.A., Professor an der Universität zu Köln, Richter am OLG Düsseldorf

Professor Dr. Oliver Fehrenbacher, Professor an der Universität Konstanz

Professor Dr. Ulrich Foerste, Professor an der Universität Osnabrück

Professor Dr. Walter Gerhardt, em. Professor an der Rheinischen Friedrich-Wilhelms-Universität Bonn

Professor Dr. Richard Giesen, Professor am Zentrum für Arbeitsbeziehungen und Arbeitsrecht (ZAAR), Ludwig-Maximilians-Universität München

Professor Dr. Dr. h.c. Wolfram Henckel, em. Professor an der Georg-August-Universität Göttingen

Professor Dr. Florian Jacoby, Professor an der Universität Bielefeld

Professor Dr. Christoph A. Kern, LL.M. (Harvard), Professor an der Ruprecht-Karls-Universität Heidelberg

Professor Dr. Peter Mankowski, Professor an der Universität Hamburg

Professorin Dr. Caroline Meller-Hannich, Professorin an der Martin-Luther-Universität Halle-Wittenberg

Professor Dr. Hans-Friedrich Müller, LL.M. (Bristol), Professor an der Universität Trier; Richter am OLG Koblenz

Professor Dr. Joachim Münch, Professor an der Georg-August-Universität Göttingen

Professor Dr. Andreas Piekenbrock, Professor an der Ruprecht-Karls-Universität Heidelberg

Professorin Dr. Nicola Preuß, Professorin an der Heinrich-Heine-Universität Düsseldorf

Professor Dr. Eberhard Schilken, em. Professor an der Rheinischen Friedrich-Wilhelms-Universität Bonn

Professorin Dr. Jessica Schmidt, LL.M. (Nottingham) Prof. an der Universität Bayreuth

Professor Dr. Peter A. Windel, Professor an der Ruhr-Universität Bochum 
